\title{
Le style des figurations paléolithiques piquetées de la vallée du Côa (Portugal) : premier essai de caractérisation ${ }^{1}$
}

\author{
Emmanuel Guy*
}

\begin{abstract}
Résumé - L'objet de ce texte consiste en une analyse stylistique menée sur plus de deux cents figurations de la vallée du Côa. Elle permet de mettre en évidence l'existence d'un style particulier fondé sur la répétition systématique de trois modes de représentation spécifiques. En outre, l'analyse montre que ce même style a été employé sur d'autres représentations paléolithiques provenant de sites distincts, parfois très éloignés de la région du Haut-Douro portugais. (0) 2000 Éditions scientifiques et médicales Elsevier SAS
\end{abstract}

\section{Art paléolithique à l'air libre / style / piquetage}

\begin{abstract}
The style of pecked paleolithic figures from Côa Valley (Portugal) : First attempt at characterization. This paper deals with a stylistic analysis made on two hundred figures from the Côa valley. It shows the existence of a specific style based on three particular ways of representation. This style is also present in other sites, of which some are very far from the portugese Haut-Douro region. (C) 2000 Éditions scientifiques et médicales Elsevier SAS
\end{abstract}

open air palaeolithic art / style / pecking

\section{Introduction}

Nous menons actuellement une recherche post-doctorale financée par la Fondation Fyssen sur les représentations paléolithiques de plein air récemment découvertes dans la vallée du Côa dans le Nord-Est du Portugal. L'art paléolithique du Côa qui s'étend de manière discontinue sur près de $20 \mathrm{~km}$ et qui compte actuellement plus de deux mille figurations connues, présente, apparemment, une certaine diversité de style. Celle-ci indique que l'activité artistique extérieure a été pratiquée dans la vallée à plusieurs moments du Paléolithique supérieur.

L'un des aspects de notre recherche consiste, précisément, à essayer d'individualiser, de manière rigoureuse, ces différentes productions figuratives encore mal connues, tant du point de vue de leur origine chrono-culturelle que des propriétés de leur expression. Ainsi, nous pouvons identifier une importante production de figures (au moins 300) réalisées par piquetage qui pré- sentent, de prime abord, une très grande unité de traitement. Ces figures offrent de surcroît d'autres caractéristiques communes qui soulignent encore le sentiment de leur parenté.

Tout d'abord leur localisation préférentielle - même si elle ne leur est pas exclusive - sur des roches situées au plus près de l'eau (par opposition à d'autres figures situées plus en hauteur sur les versants) et leur regroupement cn différents secteurs le long du fleuve (Canada do Inferno, Ribeira de Piscos, Penascosa, Quinta da Barca, Faia). De plus, ces représentations offrent la particularité fréquente d'être superposées les unes aux autres formant, dans les cas les plus extrêmes, des palimpsestes inextricables.

L'objet de ce travail consiste donc en une analyse minutieuse de l'ensemble de ces figurations piquetées, destinée à mettre en évidence les éléments précis sur lesquels reposent leur parenté ct qui fondent, selon nous, leur style commun.

\footnotetext{
${ }^{1}$ Communication présentée à la $17^{\text {ème }}$ session du Séminaire international « Représentations préhistoriques » organisé au Musée de l'Homme par le $\mathrm{P}^{\mathrm{r}} \mathrm{D}$. Vialou.

* Ethnologie préhistorique, EP 1730 CNRS, 21 allée de l'Université, F-92023 Nanterre cedex. emguy@.wae.u-paris10.fr
} 


\section{Méthodologie}

L'analyse que nous avons menée et qui a déjà été mise en cuvre à plusieurs reprises dans d'autres contextes figuratifs (Guy, 1993 ; 1998), consistc tout d'abord à rechercher certains modes de représentation dont la spécificité est susceptible, à première vue, de témoigner d'un parti pris d'expression volontaire et délibéré : tel traitement du contour, telle disproportion, telle manière particulière d'exprimer la troisième dimension, etc. La détermination de chaque trait supposé repose simultanément sur la connaissance d'autres manières paléolithiques d'exprimer la même réalité anatomique soulignant ainsi le caractère arbitraire et non « naturel » de l'élément.

Dans un second temps, il s'agit de comparer les figures entres-elles de manière à mettre en évidence la récurrence et donc l'intentionnalité du trait formel retenu et à exclure ainsi l'hypothèse d'un geste accidentel ou hasardeux.

Bien entendu, la validité d'un critère stylistique est encore affirmée par sa récurrence sur des figures provenant de sites distincts.

Ainsi, au terme de cet examen, trois modes de représentation caractéristiques du style des figures piquetées ont été mis au jour.

\section{Analyse stylistique}

\subsection{Les constructions géométriques du contour}

La première caractéristique formelle commune à l'ensemble de ces 300 fígures concerne le traitement géométrique des contours du ventre et du bord antérieur de la patte arrière systématiquement exprimés par l'intermédiaire de deux arcs d'orientation opposée (figure I). Le caractère géométrique de ce traitement est encore accentué par l'angle exagérément aigu que dessinent parfois ces deux arcs en se rejoignant au niveau de l'aine.

En outre, cette traduction en arcs opposant une courbe à une contre-courbe parait d'autant plus volontaire que sa réalisation par piquetage a nécessité plusieurs « passages » (figure 2) : un dessin préparatoire en gravure fine - généralement constitué de tracés courts multiples - que l'on voit parfois dépasser sous le piquetage à certaines intersections ou extrémités de lignes, puis, le piquetage, et, dans de nombreux cas, la régularisation du sillon piqueté ${ }^{1}$ par abrasion (surgravure).

Bicn entendu, la forme de ces contours s'inspire, dans une certaine mesure, de la réalité anatomique (figure 3) mais elle n'en est pas moins réduite à une formule purement géométrique. De fait, si ce dessin correspondait exclusivement à l'observation, on le retrouverait sur de nombreuses figures paléolithiques de styles distincts, ce qui n'est pas le cas. S'il existe, parfois, des tracés similaires sur des figures stylistiquement différentes de celles-ci, on peut penser que cette proximité relève d'une convergence fortuite due au schématisme des contours, comme le suggère une traduction beaucoup moins systématique que sur les figures du Côa.

Ainsi, nous avons regroupé plusieurs séries de lignes ventrales appartenant à des représentations d'aurochs et de chevaux (principaux sujets représentés sur les piquetages du Côa) provenant de la vallée et de différentes grottes - Lascaux (Dordogne), Ekaïn (Pays basque espagnol) et Niaux (Ariège) - que nous avons comparé à un même modèle géométrique type (cf. encadré au-dessus de chaque série) consistant en deux arcs identiques d'orientation inversée (figure 4).

Le premier constat que l'on peut tirer de cette comparaison est le respect absolu, aux seules variations d'angles près, des figures du Côa à ce modèle géométrique dont les différents exemples répètent fidèlement et systématiquement l'orientation respective des deux courbes. Le second constat est que dans tous les autres cas, au contraire, les contours se différencient de ce standard géométrique au profit d'inflexions diverses du tracé qui renvoient à des annotations anatomiques et, plus généralement, à une conception plus descriptive du contour.

Par ailleurs, il est important de considérer que d'autres parties du contour font également appel à des constructions géométriques. Ainsi, par exemple, sur les chevaux, l'extrémité de la crinière érigée dessine un bord en angle droit auquel répond, plus loin, un autre trait identique correspondant, sans doute, à la traduction simpliste d'une oreille. L'exploitation géo-

\footnotetext{
${ }^{1}$ Notons que le piquetage et la gravure sont parfois utilisés simultanément sur certaines figures en fonction des parties à dessiner : les tracés les plus rectilignes sont directement gravés, les courbes sinueuses sont d'abord piquetées
} 

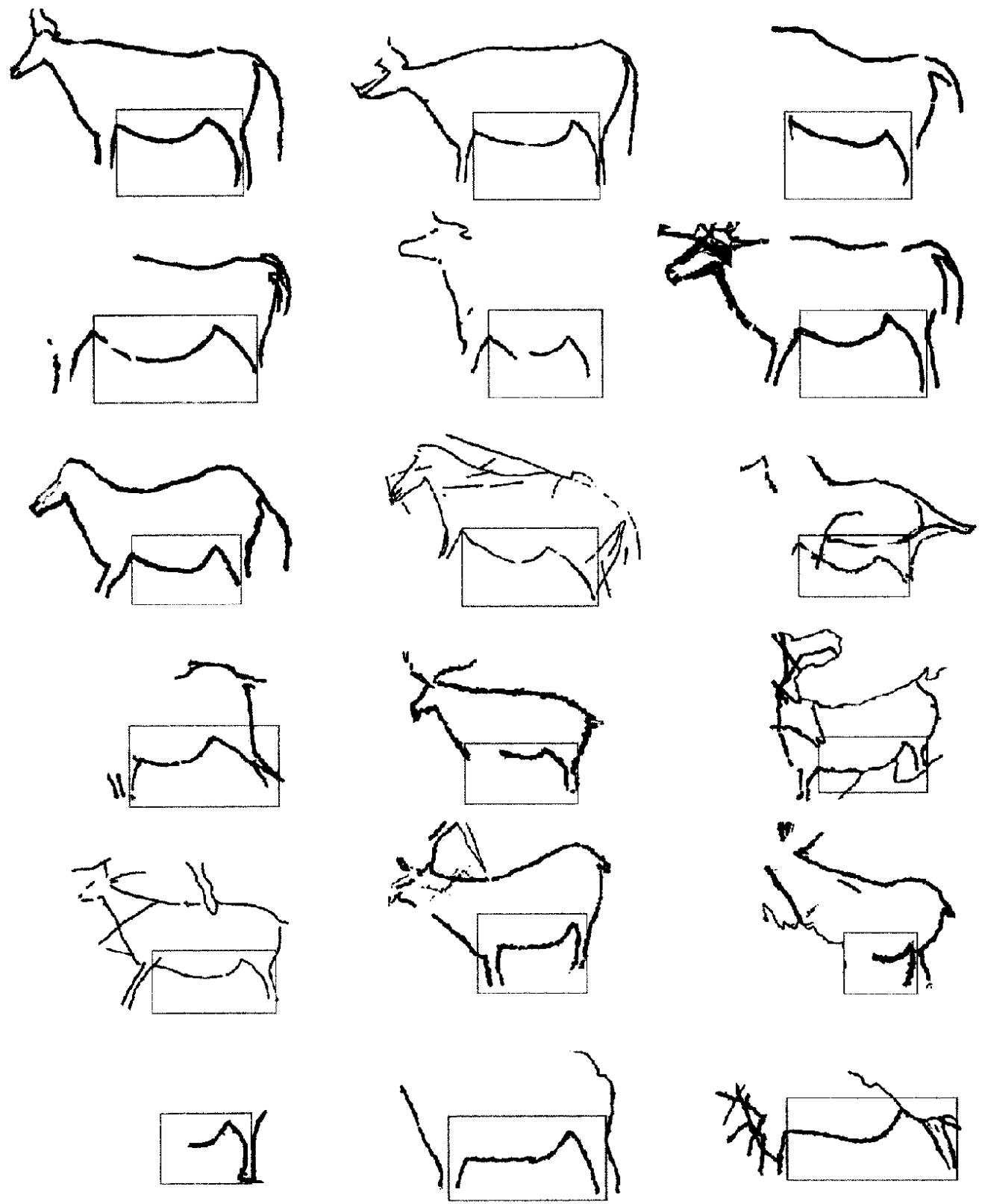

Figure 1. Le parti pris de dessin géométrique des contours du ventre et de la patte arrière des représentations piquetées de la vallée du Côa. L'orientation de certaines figures a été modifiée pour faciliter la comparaison. (relevés : d'après le Cnart).

Figure 1. The geometrical drawing bias of the outline of belley and rear paw of the dotted representations from Coa valley. The orientation of some figures has been modified in order to make the comparison casy (surveys: after the Cnart).

métrique repose ici sur le jeu de symétrie qui existe entre ces deux extrémités en angle droit systématiquement séparées par un espacc vierge (figure $5 \mathrm{~A} a$ ). Ce même dessin offre des dérivations : au lieu d'être inversés, les deux angles sont parfois opposés (figure $5 A$ b) évoquant 


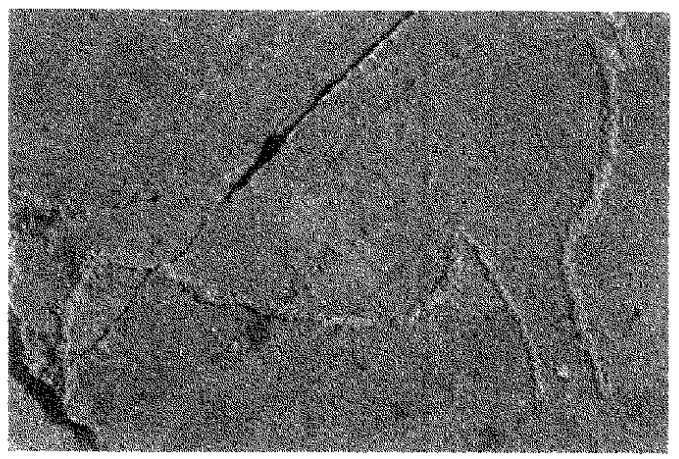

Figure 2. Détail du dessin piqueté du ventre et do la patte arrière. Ribeira de Piscos. Bouquetin. (cliché : E. Guy).

Figure 2. Detail of the pecked drawing of belley and rear paw. Ribeira de Piscos. Ibex. (Photo: E. Guy).

possiblement, tout à la fois 1'extrémité de la crinière et les deux oreilles (bien que l'on ne puisse avoir aucune certitude sur ce point, tant, précisément, le motif vaut pour lui-même et s'éloigne de toute réalité observable). Enfin, dans d'autres cas, la description se réduit à la scule interruption entre les deux parties du contour (une amorce de trait à chaque extrémité suffit à en rappeler le sens) sans autre souci de vraisemblance (figure $5 \mathrm{Ac}$ ). Cette traduction, trop fréquente pour être accidentelle est intéressante à souligner dans la mesure où elle n'hésite pas à affirmer, par son invraisemblance manifeste, le caractère purement artificicl du contour et, plus généralement, de la représentation elle-même.

La représentation de la queue des bouquetins présente, cllc aussi, une expression particulière- ment systématiséc toujours réduite à deux courts traits parallèles parfois complétés par un tracé médian (figure $5 \mathrm{~B}$ ). Enfin, on mentionnera encore le dessin de la ligne cervico-dorsale des représentations de chevaux exprimé d'une seule et même arabesque à trois courbures. L'hypothèse d'une prédétermination de cette sinuosité dans l'expression de ce tracé vient du fait que la queue cst toujours représentée dans un second temps, soit qu'elle reste ouvertement discontinue sous l'extrémité de la croupe (figure $5 \mathrm{Ca}$ ), soit qu'elle se raccorde postérieurement à elle (figure $5 \mathrm{Cb}$ ). Cette discontinuité s'explique selon nous par le fait que le tracé du contour est dicté par la succession même de ces courbes inversécs aboutissant, dès lors, à figurer postérieurement la queue dont la forme échappe nécessairement à cette alternance.

Un autre argument qui souligne la motivation d'une expression géométrique interne à ce tracé est le fait que la courbure qui marquc la croupe est généralement exagérément fcrmée, précisément, selon nous, pour répondre au jeu d'alternance avec les deux courbes qui la précède. Ce mode a pour effet sur toutes les figures de placer anormalement l'attache de la queue au niveau des fesses et non plus, comme il se doit, à hauteur de croupe.

Ces observations font échos aux propos d'A. Leroi-Gourhan sur l'automatisme du tracé cervico-dorsal caractéristique des figures de son stylc II (Leroi-Gourhan, 1965). Comme nous aurons l'occasion de le voir par la suite, les figures mentionnées par cet auteur offrent, précisément, des parentés de traitement avec ces figures piquetées de la vallée du Côa.
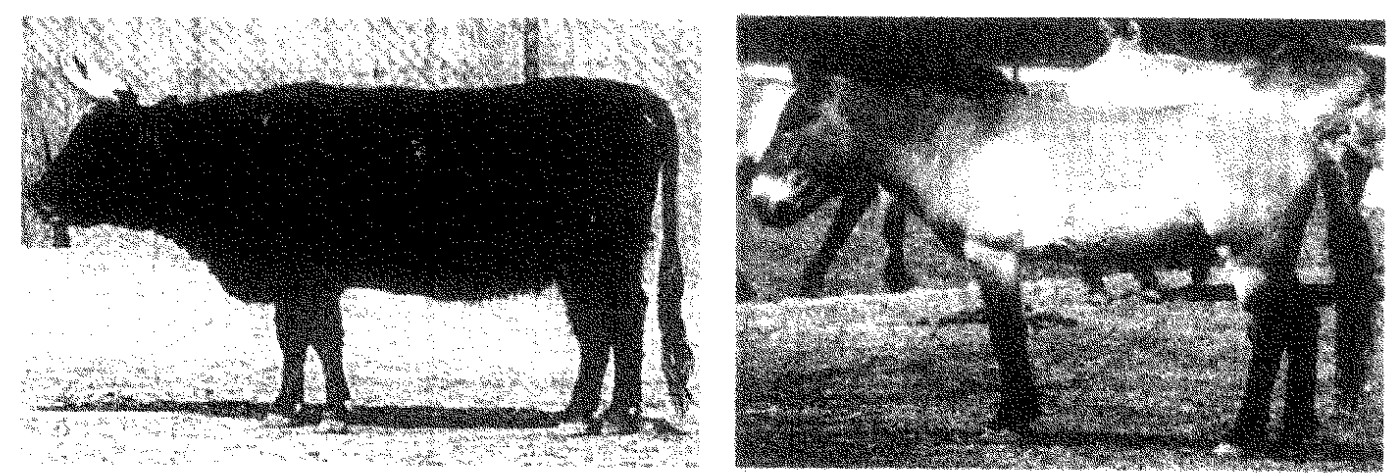

Figure 3. Aurochs et cheval de Przewalski pour comparaison (clichés : dr).

Figure 3. Bovid and Przewalski Horse for comparison (Photo: dr); 

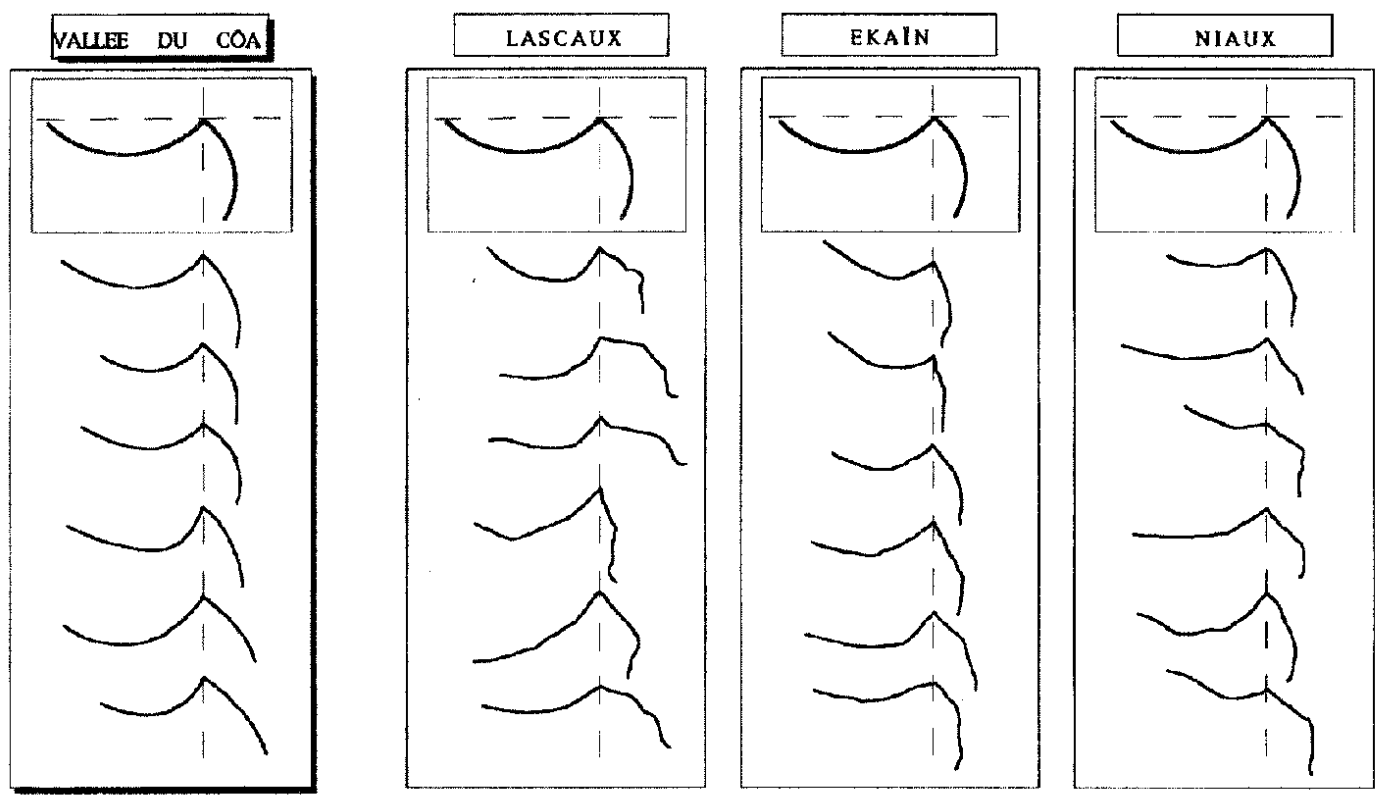

Figure 4. Comparaison des contours du ventre et de la patte arrière des figures piquetées du Côa et des grottes de Lascaux, Ekaïn et Niaux. L'orientation de certaines figures a été modifiée pour faciliter la comparaison.

Figure 4. Comparison of the outline of belley and rear paw of pecked figures from Coa and Lascaux, Ekaìn and Niaux caves. The orientation of some figures has been modified in order to make the comparison easy.

Les différentes constructions géométriques relevées mettent en évidence une conception particulière du dessin. En effet, on constate que l'expression du contour n'est pas strictement déterminée par le modèle décrit (quel que soit le degré de fidélité recherché) comme c'est habituellement le cas mais qu'elle repose aussi sur une organisation interne des tracés eux-mêmes.

\subsection{Le parti pris de représentation d'un membre unique à chaque train systématiquement dépourvu d'extrémité}

Le second élément formel caractéristique de ces représentations piquetées concerne le dessin des membres. En effet, toutes ces figures offrent la particularité d'un membre unique à chaque train systématiquement dépourvu d'extrémité (figure 6). Plus encore, la représentation des pattes se limite à deux traits parallèles sans aucun autre souci descriptif (articulation, jarret, extrémités, etc.). Cette négligence délibérée pour la traduction des membres renvoie, ici encore, à la définition que donne A. Leroi-Gourhan à propos des figures de son style II dont il note le rôle purement « accessoire » des pattes (LeroiGourhan, 1965).

\subsection{Le désintérêt volontaire à l'égard des informations anatomiques}

Le troisième trait stylistique commun à l'ensemble de ces représentations concerne le désintérêt affiché à l'égard des informations anatomiques internes (figure 7). Ainsi, on constate tout d'abord l'absence systématique sur la totalité de ces figures de toute représentation de pelage interne. Une constante que l'on ne peut attribuer à une contrainte liée au support dans la mesure où coexistent parfois sur les mêmes roches, des figures d'un style très différent qui portent un remplissage strié.

De plus, on observe que les principaux détails internes sont exprimés de manière occasionnelle et, en tout état de cause, toujours très économe : un simple point pour l'œil, un trait rectiligne horizontal pour la bouche, deux pour les naseaux, un trait vertical pour marquer le 
A

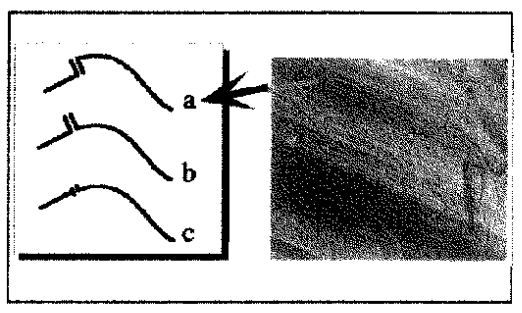

B

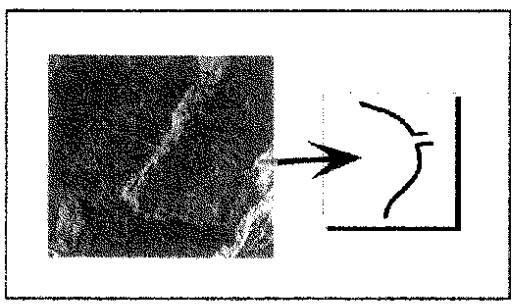

C

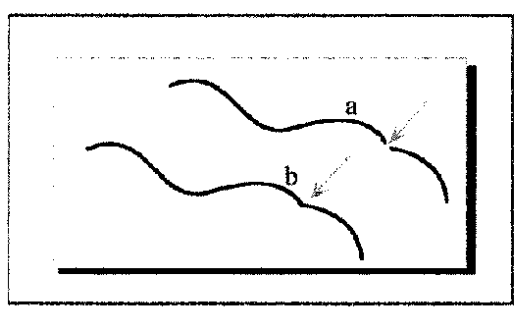

Figure 5. D'autres constructions géométriques du contour. $(\Lambda)$ le dessin de la crinière et de l'oreille des chevaux et ses dérivations; (B) le dessin de la queue des bouquetins; (C) le dessin de la ligne cervico-dorsale des chevaux.

Figure 5. Other geometrical constructions of the outline. (A) Drawing of horses' mane and ear and its derivations; (B) drawing of ibexes' tails; (C) drawing of horses' cervicodorsal line.

départ du mufle, etc. Cette pauvreté descriptive ne relève pas d'une maladresse ou d'une quelconque incapacité à voir car on note la traduction par le contour de certaines observations subtiles comme l'arrondi de la ganache sur les chevaux ou les deux bosses du garrot et des reins sur les cervico-dorsales d'aurochs. Cette sélection donne donc le sentiment d'une désinvolture délibérée à l'égard des informations anatomiques d'ordre général, au profit de quelques détails, mineurs en apparence, mais dont la présence se justifie, d'après nous, par le fait qu'elles servent plus directement à la caractérisation du sujet. Une impression que renforce le caractère invariable et systématique des mêmes annotations.

\subsection{Trois composantes répétées sur des figures provenant de sites distincts}

Il est intéressant de constater que les trois composantes formelles que nous avons relevées sur ces piquetages ont été répétées sur d'autres représentations provenant de sites distincts et $\mathrm{ce}$, indépendamment de la diversité des supports et des techniques employées (figure 8).

Il n'est pas question pour nous d'établir ici un inventaire exhaustif de ces parentés mais simplement d'évoquer quelques exemples qui soulignent ainsi la validité des trois critères mis en évidence sur les figures du Côa. C'est le cas notamment de certaines représentations pariétales des grottes d'El Reno (Espagne) d'Escoural (Portugal), de La Pileta (Espagne), de PairNon-Pair (France), de La Croze à Gontran (France) et de Mayenne-Sciences (France).

Ainsi, on constate sur toutes ces figures :

- le même dessin géométrique du ventre et de la patte arrière formant un angle aigu au niveau de l'aine. On notera que cette parenté est encore affirmée par la caractéristique commune consistant sur la plupart des figures à déporter sensiblement la convexité ventrale vers l'arrière. On rencontre, par aillcurs, de manière ponctuelle comme c'est déjà le cas à Côa - une construction identique des crinières ( $E l$ Reno, Pair-NonPair, Mayenne-Sciences) et une même discontinuité entre l'extrémité de la courbe cervicodorsale et la queue telle que nous l'avons observéc sur les chevaux piquetés du Côa;

- le même dessin systématique d'un membre unique à chaque train et dépourvu d'extrémité (avec une tentative avortée de représentation de deux antérieurs sur l'un des chevaux de la Pileta);

- la même absence (pelage) ou présence ponctuelle des principales informations anatomiques internes (œil, bouche, naseau, etc.) au profit de quelques annotations caractéristiques (ganache, bosses du garrot et des reins des cervicodorsales d'aurochs) exprimées par le contour.

\section{Conclusion et perspectives}

Nous avons mis en évidence, au cours de cette analyse, l'existence d'un style caractérisé par trois modes particuliers observés sur plus de trois cents piquetages de la vallée du Côa.

S'il existe ici une adéquation manifeste entre le style et la technique d'exécution employée - 

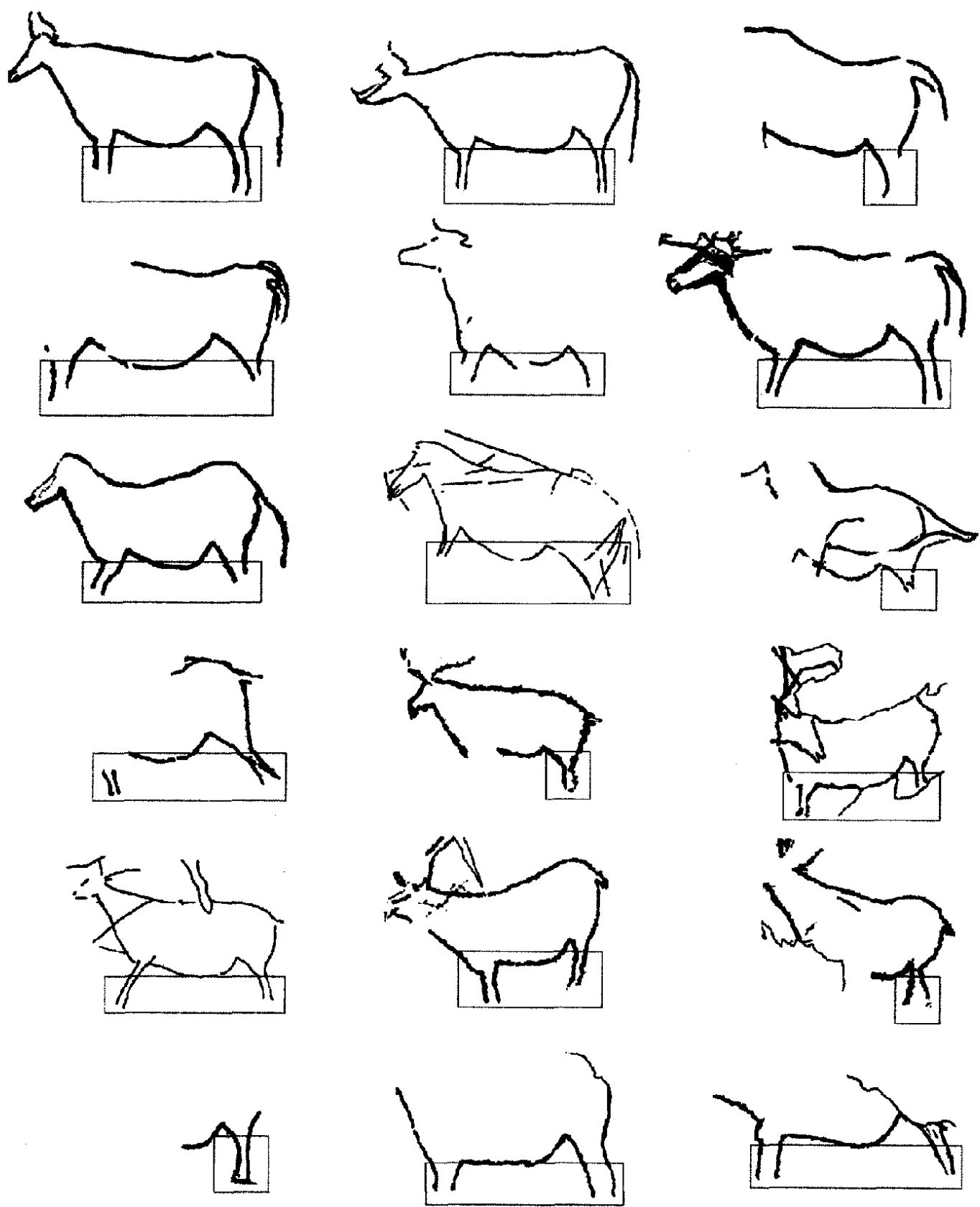

Figure 6. Le parti pris de dessin d'un membre unique et dépourvu d'extrémité à chaque train. L'orientation de certaines figures a été modifiée pour faciliter la comparaison. (relevés : d'après le Cnart).

Figure 6. Drawing bias of a unique member without any extremity. The orientation of some figures has been modified in order to make the comparison easy (surveys: after the Cnart).

il existe très peu de figures simplement gravées se rapportant à ce style -, il existe également à Côa d'autres piquetages, en faible nombre, qui ne répondent pas ou incomplètement à ces trois critères stylistiques (on peut citer par exemple, les bouquetins de la roche 3 de Quinta da Barca, 

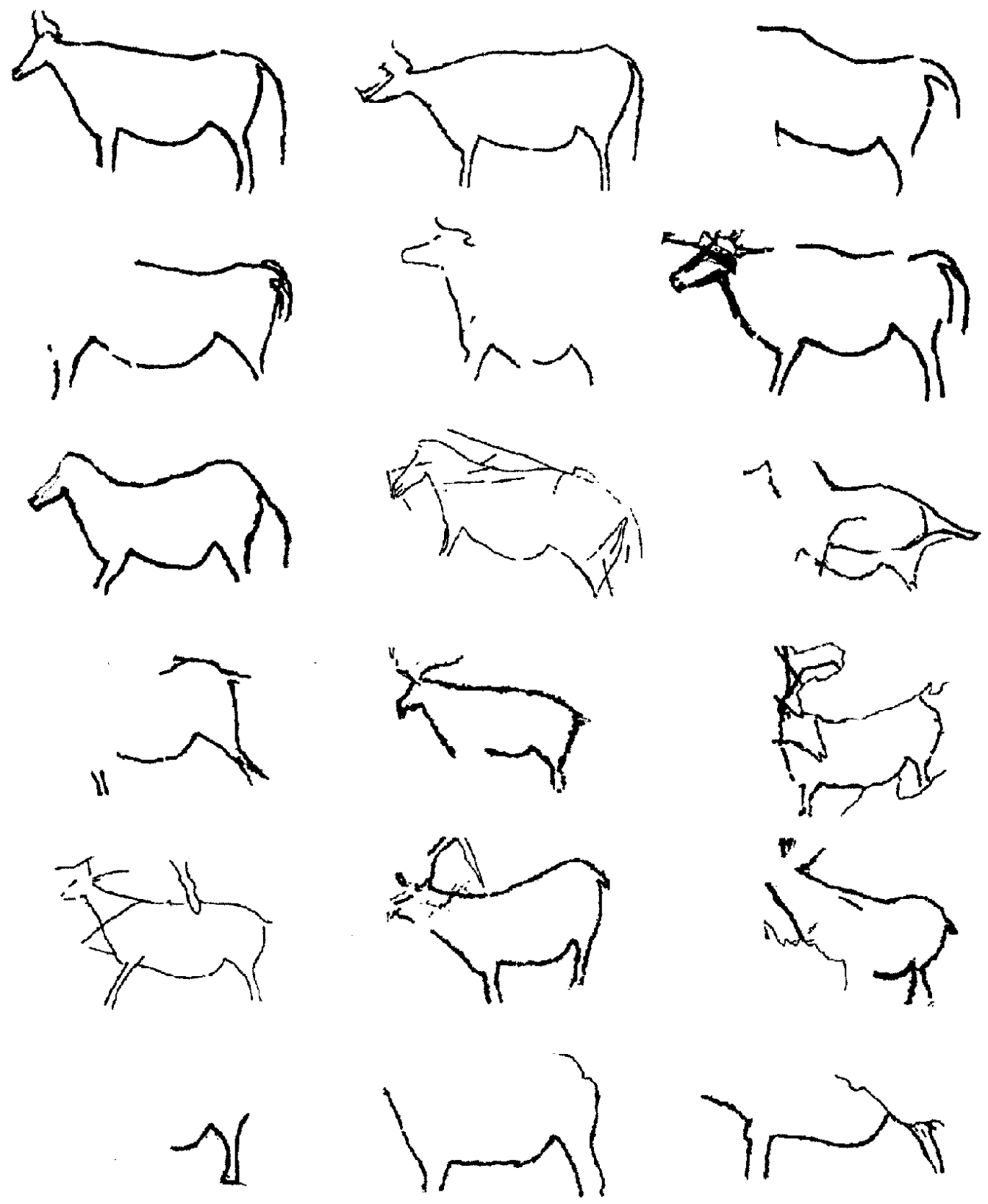

Figure 7. Le désintérêt volontaire à l'égard des principales informations anatomiques des représentations piquetées de la vallée du Côa. L'orientation de certaines figures a été modifiée pour faciliter la comparaison. (relevés : d'après le Cnart).

Figure 7. Voluntary disinterest towards main anatomical information of pecked representations from Côa valley. The orientation of some figures has been modified in order to make the comparison easy (surveys: after the Cnart).

le cheval de gauche de la roche 14 de Canada do Inferno, le bouquetin du panncau 1 de Rego da Vide, etc.). On est en droit de penser que ces dif- férences ne relèvent pas de simples variations d'exécution individuelle mais bien de conception stylistiques autres, dans la mesure où, comme 

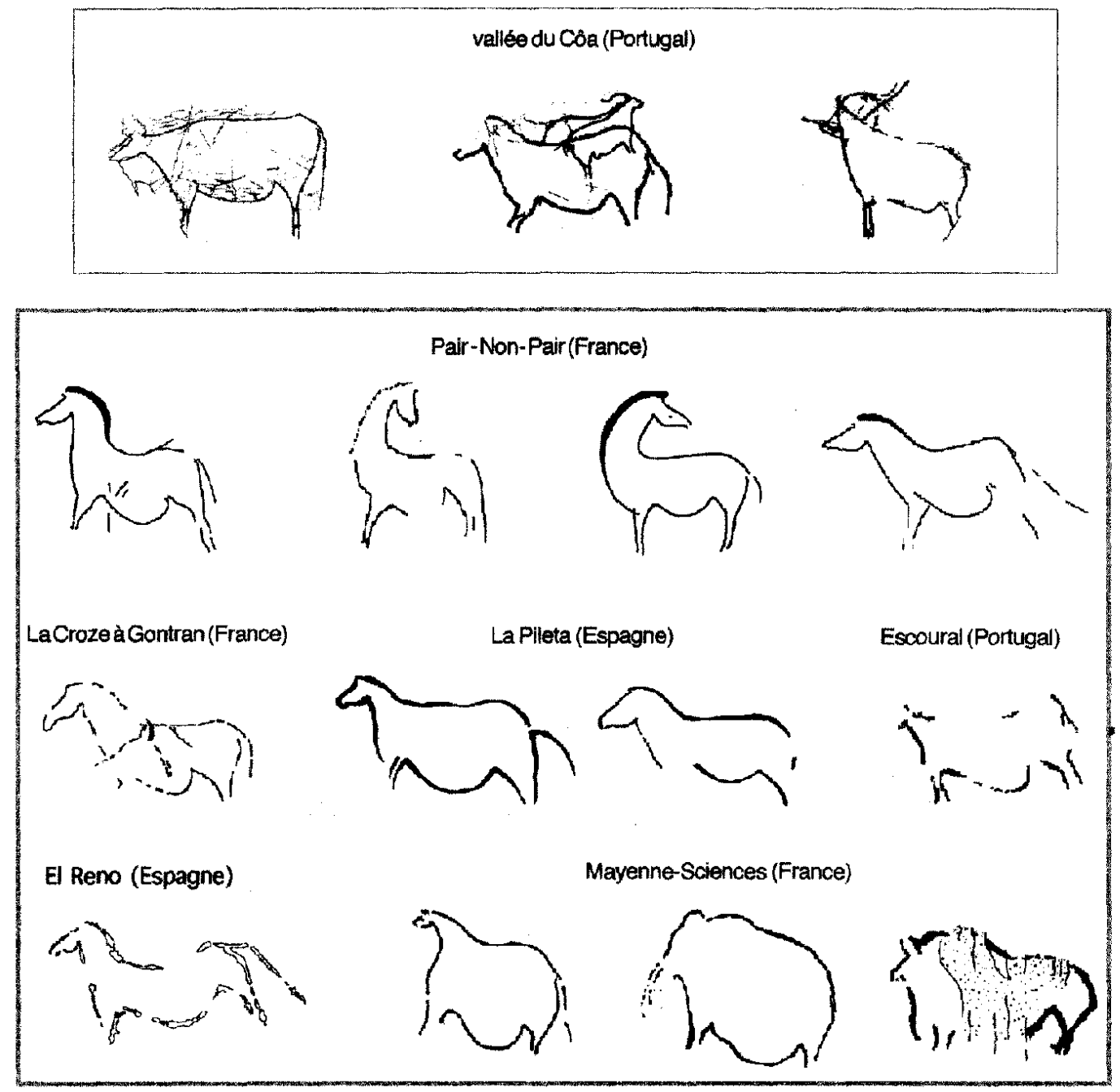

Figure 8. Comparaison entre les figures du Côa et divers exemples pariétaux offrant les mêmes caractères formels relevés. L'orientation des figures a été modifiée pour faciliter la comparaison. (relevés: Foz Côa, d'après le Cnart ; El Reno, d'après Alcolea Gonzalez et alii., Escoural, d'après Lejeune; la Pileta, d'après Breuil ; Pair-Non-Pair et La Croze à Gontran, d'après Delluc ; Mayenne-Sciences, d'après Bouillon).

Figure 8. Comparison between the Côa's figures and various rock art examples showing the same formal characters. The orientation of some figures has been modified in order to make the comparison easy (surveys: Foz Côa after the Cnart; El Reno after Alcolea Gonzalez et al.; Escoural after Lejeune; La Pileta after Breuil; Pair non Pair and La Croze à Gontran after Delluc; Mayenne-Sciences after Bouillon).

on l'a vu, les trois modes formels relevés se répètent aussi sur des figures provenant de sites distincts.

En outre, signalons que l'unité stylistique de la production analysée ici s'est vue, en quelque sorte, confirmée par la découverte récente (décembre 1999) d'un nouveau panneau portant des figures piquetces de même style en contact avec des niveaux d'occupation. Les recouvrcments indiquent que ces figures ont été réalisées à une même époque, sans doute au Protosolutréen, soit, vers $21500 \mathrm{BP}$.

Les trois modes de représentation mis en ćvidence témoignent d'un véritable parti pris de 


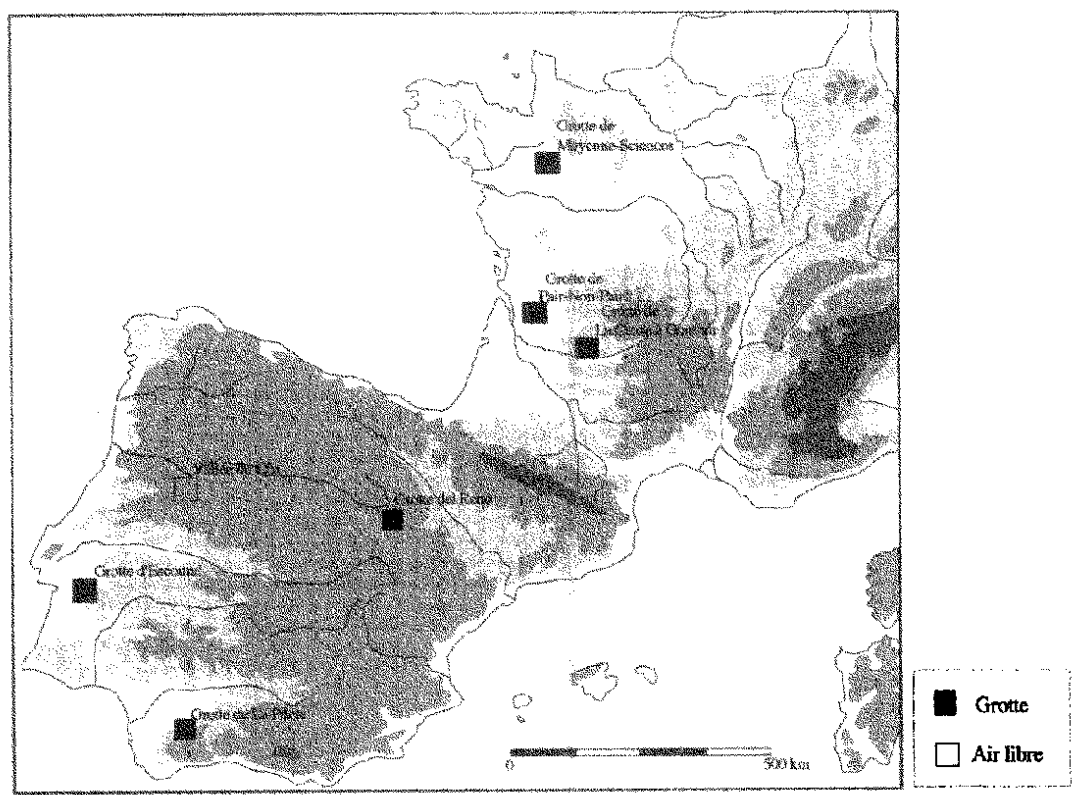

Figure 9. Carte de répartition des sites mentionnés au cours de l'analyse.

Figure 9. Distribution map of cited sites during the analysis.

schématisme. En effet, on constate que la conception de ces figures tourne délibérément le dos à l'observation au profit d'un intérêt porté sur la seule caractérisation des sujets (aurochs, cheval, bouquetin, etc.). Ainsi, comme on l'a vue, l'expression du contour n'est pas strictement déterminée par le modèle décrit, mais elle repose sur des agencements internes qui standardisent différentes parties de la figure.

De plus, on constate que tous les éléments qui ne participent pas expressément à la caractérisation de l'animal comme les membres ou les informations anatomiques d'ordre plus général que sont le pelage, l'wil, la bouche, etc. sont délibérément négligés voirc purement et simplement abandonnés. Il ne faut done pas concevoir la pauvreté descriptive de ces figures comme le résultat d'une maladresse ou d'un défaut d'observation mais, au contraire, comme un véritable choix d'expression conforme aux normes visuelles définies par une culture particulière.

Bien entendu, il ne s'agit là que d'une première exploration qui devra être étayée ultéricurement par des observations supplémentaires destinées à affiner encore notre lecture et à préciser le sens des orientations formelles de ce langage.
Par ailleurs, cette analyse a montré que ces trois conventions stylistiques se répétaient sur des figures provenant de sites distincts, parfois très éloignés de la région considérée (figure 9). C'est le cas des grottes de Pair-Non-Pair et de La Croze à Gontran situées dans le sud-ouest à plus de $700 \mathrm{~km}$ de distance en ligne droite de la région considérée et, plus encore, de la grotte de Mayenne-Sciences distante d'environ $1000 \mathrm{~km}$ en ligne droite.

La diffusion de ces procédés stylistiques d'un site à l'autre ne fait guère de doute. En effet, l'hypothèse d'une convergence fortuite entre ces figures paraît d'autant moins probable que l'on n'a pas affaire à la répétition d'un simple trait isolé mais à l'association systématique de trois éléments particuliers qui constituent une structure d'expression conventionnelle. Une diffusion à une telle échelle géographique soulève plusieurs questions que nous n'aborderons pas ici compte tenu du caractère encore préliminaire de ces rapprochements. Une prochaine étude aura précisément pour objectif d'établir une cartographie la plus exhaustive possible des figurations se rapportant à ce même style. En revanche, on notera d'ores et déjà que ces correspondances entre sites témoignent d'une contemporanéité vraisemblable entre l'activité 


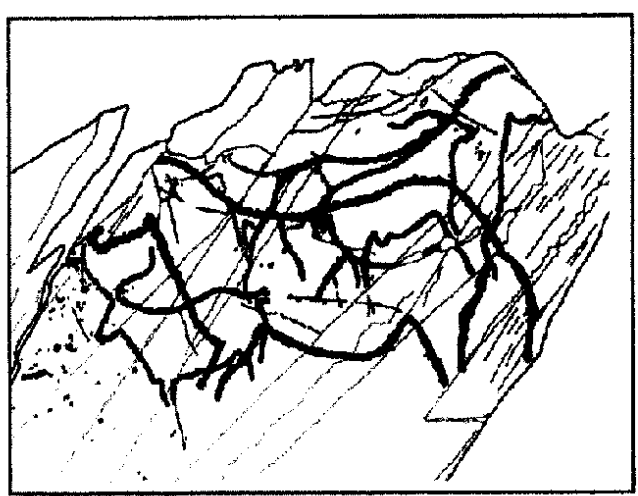

A

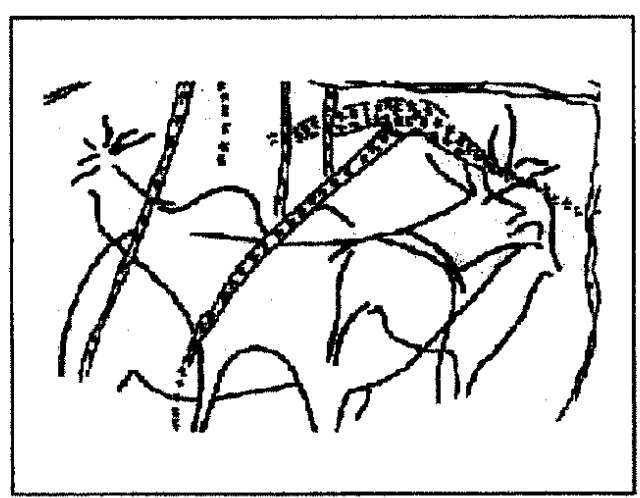

B

Figure 10. Comparaison entre l'assemblage des figures d'un panneau (Roche 6) de la vallée du Côa à Penascosa $(\Lambda$ ) et un panneau de la grotte de Pair-Non-Pair (B). (Relevés: vallée du Côa, d'après le Cnart ; Pair-Non-Pair, d'après Delluc.)

Figure 10. Comparison between the fïgures assemblage of a pannel (Rock 6) from Côa valley at Penascosa (A) and of a panncl from Pair non Pair cave; (B) (Surveys: Côa valley after the Cnart; Pair non Pair after Delluc.)

figurative pratiquée à l'air libre et en grotte. Mais cette unité d'expression ne signifie pas pour autant que ces deux contextes aient nécessairement eu la même fonction symbolique.

L'enjeu d'un approfondissement de ces comparaisons entre sites consistera, à tenter de préciser la nature des relations entre ces contextes. On perçoit déjà, ici ou là, quelques parentés dans l'assemblage des figures (figure 10). Ainsi, par exemple, à Côa, un panneau du site de Penascosa (Roche 6) présente deux chevaux superposés en sens inverse (A). Le cheval en profil droit porte un bouquetin luimême adossé à un autre bouquctin de petite dimension. Le cheval en profil inverse porte un autre bouquetin placé sous son encolure. On retrouve un dispositif partiellement comparable sur l'un des panneaux de Pair-Non-Pair (B) consistant, notamment, en deux cervidés superposés en profil inversés (un cerf et un mégacéros) avec, ici encore, un bouquetin placé sous l'encolure de l'une des figures (le cerf).

Mais il existe aussi des différences. Ainsi, le parti pris d'accumulation des figures, les unes sur les autres semble être une exclusivité de ces représentations de plein air. On rencontre, certes, à Pair-Non-Pair, Escoural ou El Reno des cas de figures superposées les unes aux autres. Mais jamais on ne retrouve sur ces exemples ce même principe consistant à surgraver «à l'infini » les figures sur une même surface de la roche. $\grave{A}$ ce titre, il sera sans doute instructif d'élargir nos observations aux représentations extérieures des nombreux abris ou grottes de la vallée du Nalón (La Lluera, La Viña, Santo Adriano, Los Torneiros, etc.) située dans les Asturies, au nord du Côa, ainsi que de certaines grottes de la vallée de l'Ardèche (Le Figuier, Huchard, Chabot). En effet, toutes ces figures paraissent présenter plusieurs caractéristiques communes avec les piquetages du Côa analysés : elles sont très profondément incisées dans la roche, elles sont fréquemment enchevêtrées, elles sont situćcs en contact ou à proximité de la zone d'habitat et enfin, elles sont observables à la lumière du jour.

\section{Remerciements}

Je remercie D. Vialou de son invitation à participer au séminaire du Musée de l'Homme qu'il anime. Je suis particulièrement reconnaissant à la Fondation Fyssen de son soutien. Enfin, je remercic J. Zilhão, Directeur de l'Institut portugais d'archéologie ainsi que A. Baptista et l'équipe du Centre national d'art rupestre portugais de leur confiance.

\section{Bibliographie}

Alcolea Gonzalez, J.J., Balbin Berhmann, R. de., Garcia Valero, M.A., Jimenez Sanz, P.J., 1997. Nuevos descubrimientos de arte rupestre paleo- 
lítico en el centro de la Penisula Ibérica : La Cueva del Reno (Valdesoto, Guadalajara), In : Il Congresso de arquelologia peninsular, In : Balbin Behrman, R. de et P. Bueno, Eds., Paleolitico y epipaléolitico, Zamora, 1996.

Baptista, A.M., 1999. No tempo sem tempo. A arte dos caçadores paleoliticos do Vale do Côa, Ed. Parque Archeológico Vale do Côa.

Bouillon, R., 1984. Grotte Mayenne-Sciences. L'art des cavernes, Atlas des grottes ornées paléolithiques françaises, Ministère de la Culture, Paris, pp. 567-571.

Delluc, B. et G., 1991. L'art pariétal archaïque en Aquitaine, XXVIII ${ }^{e}$ supplément à Gallia Préhistoire, CNRS Éd., Paris.

Guy, E., 1993. Enquête stylistique sur l'expression figurative épipaléolithique en France : de la forme au concept. Paleo 5, 333-373.

Guy, E., 1998. Évolution des formes dans l'art figuratif paléolithique occidental. Introduction à une grammaire stylistique. Thèse de Doctorat de l'Université de Paris I, 1 vol.

Lejeune, M., 1996. L'art pariétal de la grotte d'Escoural (Portugal) : analyse critiquc, comparaisons et problèmes. In : Recherches Préhistoriques à la grotte d'Escoural, Liège, Eraul 65, 137-240.

Leroi-Gourhan, A., 1965. Préhistoire de l'art occidental, Mazenod Éd., Paris.

Sanchidrian Torti, J.L., 1997. Propuesta de la secuencia figurativa en la Cueva de la Pileta. In : El Món mediterrani deprés del Pleniglacial (18 000-12 000 BP), (J.M. Fullola \& N. Soler, Eds.), Sèrie Monogràfica, 17, Museu d'Arqueologia de Catalunya, Girona, pp. 411-430.

Zilhão, J. (ed.), 1997. Arte rupestre e pré-história do Vale do Côa, Trabalhos de 1995-1996. Relatório científico ao governo da República Portugucsa claborado nos termos da resoluçao do Conselho de Ministros 4 (96), de 17 de Janeiro, Lisboa, Ministério da Cultura. 